\title{
Exploring the impact of elevated depressive symptoms on the ability of a tailored asthma intervention to improve medication adherence among urban adolescents with asthma
}

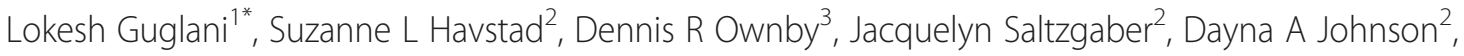

Christine C Johnson ${ }^{2}$ and Christine LM Joseph ${ }^{2}$

\begin{abstract}
Background: In patients with asthma, medication adherence is a voluntary behavior that can be affected by numerous factors. Depression is an important co-morbidity in adolescents with asthma that may significantly impact their controller medication adherence and other asthma-related outcomes. The modifying effect of depressive symptoms on an asthma intervention's ability to improve asthma controller medication adherence among urban adolescents with asthma has not yet been reported.

Objective: To assess self-reported symptoms of depression as an effect modifier of the relationship between randomization group and controller medication adherence at 6-month follow-up.

Methods: These analyses use data from a randomized controlled trial (RCT) conducted in Detroit high schools to evaluate a tailored asthma management program. The intervention included referrals to school or community resources for students reporting symptoms of depression and other issues. "Elevated depressive symptoms" was defined as a positive answer to $\geq 5$ of 7 questions from a validated tool included on the baseline questionnaire. Self-reported adherence to controller medication was collected at intervention onset (session 1) and at 6-month follow up. Analyses were restricted to students with report of a controller medication at baseline. Logistic regression was used to assess elevated depressive symptoms as an effect modifier of the relationship between randomization group and 6-month adherence.

Results: Of the 422 students enrolled in the RCT, a controller medication was reported at intervention onset by $\mathrm{n}=123$ adolescents (29\%). Analyzing this group, we observed an interaction between elevated depressive symptoms and adherence $(p=0.073)$. Stratified analysis showed better adherence in treatment group adolescents meeting criteria for elevated depressive symptoms at baseline as compared to the control group (adjusted Odds Ratio [aOR] = 9.50; $p=0.024)$. For adolescents without elevated depressive symptoms at baseline, differences in adherence by group assignment did not reach statistical significance (aOR 1.40, $p=0.49$ ).

(Continued on next page)
\end{abstract}

\footnotetext{
* Correspondence: Iguglani@med.wayne.edu

'Pediatric Pulmonary Division, Department of Pediatrics, Children's Hospital of Michigan, Wayne State University School of Medicine, 3901 Beaubien St, Detroit, Ml 48201, USA

Full list of author information is available at the end of the article
} 
(Continued from previous page)

Conclusions: In this sample of students reporting controller medications at baseline, report of elevated depressive symptoms at baseline and randomization to the intervention group was associated with significantly better adherence at 6-month follow up when compared to that of a control group. Larger studies are needed to evaluate the impact of depression on the relationship between adherence and asthma intervention effectiveness.

Keywords: Asthma, Depression, Medication adherence, Randomized controlled trial, Self-management, Adolescents, Urban

\section{Background}

There is a significantly higher prevalence of asthma in urban African American and Latino adolescents and these groups are known to have worse asthma-related outcomes than their White counterparts [1]. Asthma control is impacted by a number of factors, including adherence to prescribed regimens. Adherence, as per its definition, is an "active, voluntary and collaborative involvement of the patient in a mutually acceptable course of behavior to produce a therapeutic result". Adherence is influenced by a number of internal and external factors including patient beliefs and attitudes, disease and therapy-related factors, health system characteristics, and socioeconomic factors [2]. According to the literature, urban adolescents with asthma in general have poor adherence to asthma controller medications [3]. Studies using electronic monitoring of controller medication adherence in adolescents [4,5] have shown 40-50\% adherence, with significantly lower rates of adherence in African American adolescents [6].

Depression is a known co-morbidity of asthma; however, few studies provide estimates of depression as co-morbidity in adolescents with asthma. Existing reports suggest the prevalence of depression among adolescents with asthma ranges from 7.2 to $16.3 \%$ [7-9]. Depression may impact quality of life in adolescents with asthma. In a previous analysis of Puff City data, we have shown that depressive symptoms significantly impact emotional quality of life [10]. Depression has been associated with medication adherence in adults and in diseases other than asthma [11-13], but the impact of depression on asthma intervention effectiveness with regard to controller medication adherence has not been explored in urban adolescents.

The present analyses explore the impact of elevated depressive symptoms at baseline on the ability of an asthma intervention to improve adherence to controller medications at 6-month follow-up, among urban teens with asthma. The intervention upon which these analyses are based is Puff City, a computer-tailored, web-based program for urban teens with asthma, originally developed and tested in 2001 [14]. An enhanced version was evaluated in Detroit high schools using a randomized controlled trial conducted from 2007-2011 [15]. The subgroup analyses reported here include urban teenagers that were enrolled in the 2007 - 2011 RCT of Puff City, and reported controller medication(s) at intervention onset.

\section{Methods}

The details of the Puff City randomized controlled trial have been published previously [14-16]. Briefly, to identify students with asthma or asthma symptoms, caregivers of all 9th through 12th grade students of six Detroit public high schools were notified by mail of a Lung Health Questionnaire (LHQ) to be administered during an English class. Parents could opt out of having their child complete the LHQ by signing and returning the letter to the school or by contacting the school. Eligibility to participate in the RCT was based on LHQ responses. To be eligible, the students had to have a physician diagnosis of asthma accompanied by one or more of the following: presence of daytime and/or nighttime symptoms in the last 30 days, medication use for asthma symptoms in the last 30 days, and $\geq 1$ refill(s) of beta-agonists in the last 1 year. Adolescents without a physician diagnosis of asthma were also eligible to participate if they had positive responses to items selected from the International Study of Asthma and Allergies in Childhood (ISAAC) [17], and had symptom frequencies similar to those used in EPR 2 and 3 (e.g., for "mild persistent asthma" criteria from EPR3 include "symptoms $\geq 2$ days/ week, nighttime awakening $\geq 3-4 x /$ month, and interference with normal activities $=$ minor limitation") $[18,19]$. Students identified as eligible for the RCT based on the above criteria were mailed an invitation to participate in the RCT, along with forms for parental written consent and student written assent. Once consent and assent were obtained, a study ID was assigned and entered into the study database. In this way, no student data was shared with investigators until appropriate informed consent was obtained [15]. After a baseline assessment, consenting students were randomized into a treatment or control group (Figure 1). The treatment group underwent a total of 4 computer-based tailored online asthma management sessions that featured topics such as asthma management behaviors (including asthma medication use and adherence, having a rescue inhaler nearby, and smoking cessation and/or reduction), trigger avoidance 


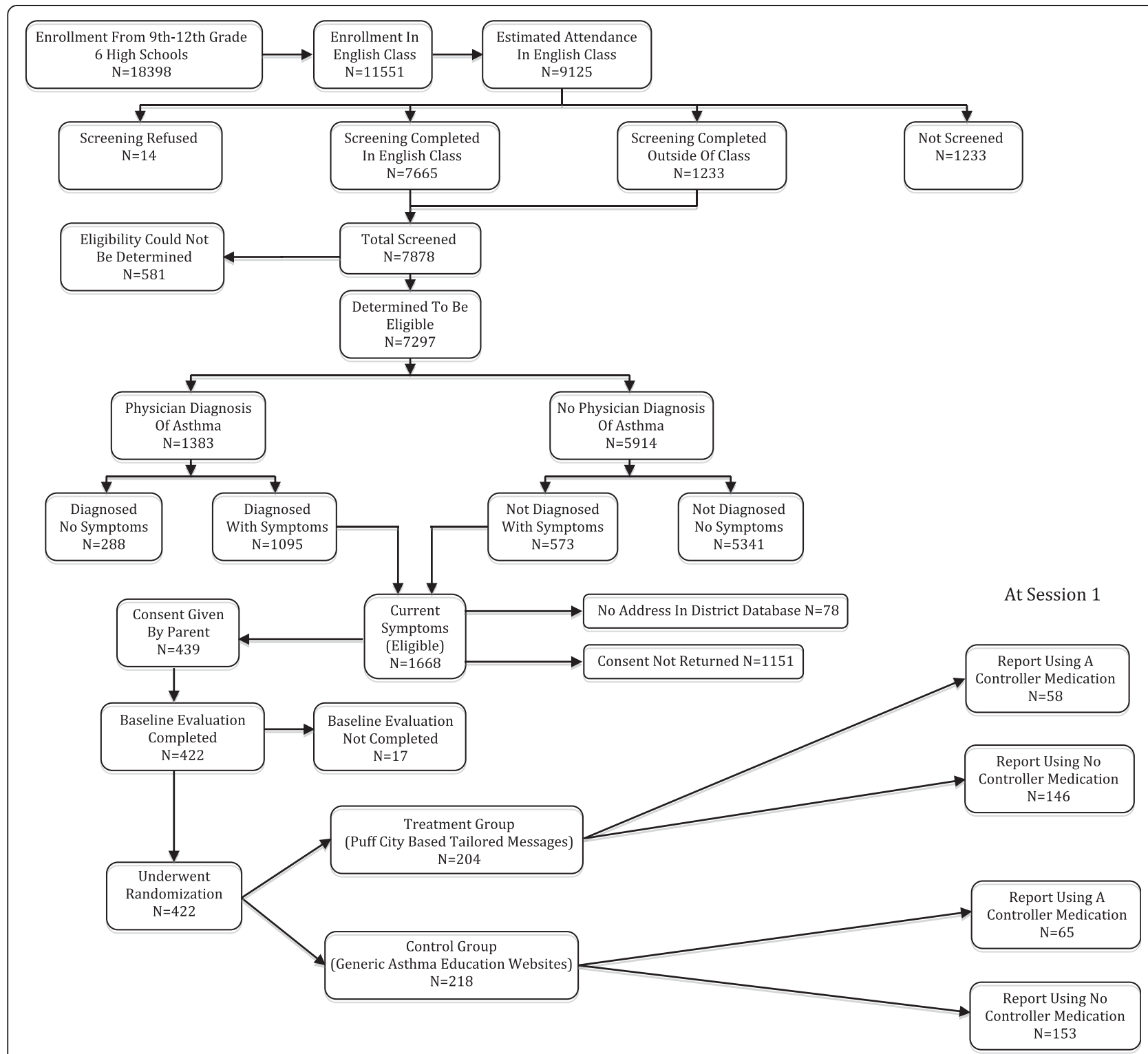

Figure 1 CONSORT Flow diagram for the school-based Puff City randomized controlled trial showing the screening of participants and breakdown of treatment and control groups.

and basic asthma physiology. The control group was provided access to existing, generic asthma education websites during 4 computer sessions of duration similar to that of the treatment group. Asthma controller medications that were prescribed by a physician were requested from both treatment and control group students at the onset of intervention session 1 using a medication selection module designed for this purpose. The module displayed pictures of medications listed in the Health plan Employer Data and Information Set (HEDIS) measure for asthma called Use of Appropriate Medications for People with Asthma [20], and included corticosteroids, inhaled steroid combinations, leukotriene receptor antagonists, mast cell stabilizers, and antibody inhibitors. Participants were asked to select the asthma medications they were currently taking, if any. All medications reported by the participant were categorized as "controller" or "rescue".

A referral coordinator was also part of the intervention. The referral coordinator's task was to assess, refer, and follow-up with students in the intervention group identified to be at-risk of a serious event through a risk assessment report generated by the data management system [16]. Students were contacted if they reported sharing asthma medication, severe asthma symptoms, lack of physician or health insurance, and/or depressive symptoms. Treatment group students with depressive symptoms were usually referred to school-based resources (e.g., 
school counselor, school social worker, or school-based clinic); and to community-based resources when school resources were not available.

As part of the study protocol, participants received mail and telephone reminders to login to the program and complete a follow-up survey scheduled for 6-months post-baseline. Survey questions collected information on asthma outcomes (e.g., symptom-days, symptom-nights, days of restricted activity), as well as information on controller medication adherence. Follow-up questions were the same for treatment and control group students, although treatment group students could receive additional "booster" messages based on responses to the 6month survey questions. This study was approved by the Institutional Review Boards of the participating institutions (IRB Protocol \#4579) and by the Detroit Public Schools Office of Research, Evaluation and Assessment.

\section{Study definitions}

Depressive symptoms were reported at baseline using 7 questions from the Diagnostic Predictive Scale (DPS) that enquires about symptoms typically associated with depression in the preceding 6-months [21]. The DPS is a result of adaptations to the Diagnostic Interview Schedule for Children (DISC), which is a structured diagnostic instrument specifically designed for use by non-clinicians [22]. The fourth version (DISC-IV) was further adapted to create several shorter scales (including the Diagnostic Predictive Scale used in this study) for use as screening tools for various psychiatric diagnoses, including depression [21]. The DPS has been tested in various populations and has been reported to be an efficient and reliable screening tool $[23,24]$ for children between the ages of 8 to 18 years. Using published cutoff scores established by Lucas et al. [21], elevated depressive symptoms was defined as a positive response to 5 or more questions on the DPS. Adherence to asthma controller medication was defined as self-reported use of the medication on 5 or more days out of last 7 days. Controller medication adherence collected at the 6-month follow-up was the primary outcome of these analyses.

\section{Statistical analysis}

Since the goal of these analyses was to assess the effect of depression on the ability of the intervention to improve controller medication adherence, analyses were restricted to the group of adolescents reporting asthma controller medications at baseline. Logistic regression was used to assess elevated depressive symptoms as an effect modifier of the relationship between randomization group and controller medication adherence at 6-months using the $\mathrm{p}$ value of $<0.10$ as indicating the presence of effect modification and the need to present stratum-specific results [25]. Baseline controller medication adherence was included as a covariate in all logistic regression models. Adjusted odds ratios (aOR) and corresponding 95\% confidence intervals were calculated to describe the association between randomization group and controller medication adherence at 6-months.

\section{Results}

The breakdown of the study population is shown in Figure 1. Baseline assessment was completed by 422 adolescents. A total of 58 adolescents in the treatment group (28.4\%) and 65 in the control group (29.8\%) reported a controller medication at the start of the intervention (Table 1). Among those reporting use of a controller medication at intervention onset, the percentage of adolescents in the treatment and control groups meeting criteria for elevated depressive symptoms was $20.7 \%$ $(\mathrm{n}=12)$ and $24.6 \%(\mathrm{n}=16)$, respectively, $\mathrm{p}=0.60$. Controller medication adherence for treatment and control group students at intervention onset was $24.1 \%(\mathrm{n}=14)$ and $27.7 \%(\mathrm{n}=18)$ respectively.

At the 6-month follow up, after adjusting for baseline adherence, 22 adolescents in the treatment group (37.9\%) reported controller medication adherence, as compared to 17 adolescents in the control group (26.2\%) (Table 2). The relationship between elevated depressive symptoms at baseline and controller medication adherence met criterion for the presence of an interaction $(\mathrm{p}=0.073)$ $[16,25]$. Stratified analysis is presented in Table 3. For adolescents that reported elevated depressive symptoms at baseline, 7/12 (58.3\%) in the treatment group reported being adherent to their controller medication at the 6-month follow-up, while 2/16 (12.5\%) were adherent in the control group, $\mathrm{aOR}=9.5 ; \mathrm{p}=0.024$. For adolescents that did not report depressive symptoms at baseline, medication adherence at 6-month follow-up was only slightly higher among students randomized to the treatment

Table 1 Prevalence of elevated depressive symptoms and controller medication adherence at intervention onset (Session 1) for teens in the treatment and control groups

\begin{tabular}{|c|c|c|c|c|c|c|}
\hline \multirow[b]{2}{*}{$\%$ of teens with elevated depressive symptoms at baseline $(n)$} & \multicolumn{2}{|c|}{ Treatment $\mathbf{N}=58$} & \multicolumn{2}{|c|}{ Control N = 65} & \multirow{2}{*}{$\begin{array}{c}\text { Odds ratio }(\mathbf{9 5 \%} \mathbf{C l}) \\
0.80(0.34,1.87)\end{array}$} & \multirow{2}{*}{$\frac{p \text { value }}{0.60}$} \\
\hline & 20.7 & $(12)$ & 24.6 & $(16)$ & & \\
\hline \multicolumn{7}{|l|}{ Core behavior and report of medication at session 1} \\
\hline Controller medication, adherent $\geq 5$ of last 7 days & 24.1 & (14) & 27.7 & $(18)$ & $0.83(0.37,1.87)$ & 0.65 \\
\hline Controller medication, not adherent $<5$ of last 7 days & 75.9 & (44) & 72.3 & $(47)$ & & \\
\hline
\end{tabular}


Table 2 Comparison of controller medication adherence at 6 month follow-up for by randomization group for students included in the analysis sample*

\begin{tabular}{|c|c|c|c|c|c|c|}
\hline Core behavior at 6 months** & \multicolumn{2}{|c|}{ Treatment $\mathrm{N}=58$} & \multicolumn{2}{|c|}{ Control $\mathrm{N}=65$} & Adjusted ${ }^{* *}$ odds ratio $(95 \% \mathrm{Cl})$ & $p$ value \\
\hline Controller medication, adherent $\geq 5$ of last 7 days & 37.9 & $(22)$ & 26.2 & $(17)$ & $2.10(0.89,4.92)$ & 0.089 \\
\hline Controller medication, not adherent $<5$ of last 7 days & 62.1 & $(36)$ & 73.8 & $(48)$ & & \\
\hline
\end{tabular}

*Students enrolled in Puff City and reporting a controller medication at baseline assessment. **Adjusted for controller medication adherence at start of intervention.

group (32.6\%) compared to the controls $(30.6 \%)$ at the 6 -month follow up, aOR $=1.4 ; \mathrm{p}=0.49$.

\section{Discussion}

The Puff City program uses tailoring to promote positive behaviors such as regular use of controller medications by providing personalized health messages to help address the adolescents' beliefs, attitudes and barriers to behavior change in addition to referrals from an asthma referral coordinator. Results of these subgroup analyses suggest that the effectiveness of a program to improve adherence to controller medications in urban adolescents with asthma may be significantly impacted by the presence of depressive symptoms. For this reason, it may be worthwhile to address depressive symptoms when treating asthma in order to improve asthma-related outcomes. We did note a modicum of improvement in medication adherence among treatment group students who did not meet criteria for elevated depression at baseline, but a comparison to the control group did not reach statistical significance. Therefore, interventions to improve controller medication adherence in adolescents with and without depressive symptoms may still be needed. Our results may have important implications for designing future interventions specifically targeting improvements in controller medication adherence in urban adolescents with asthma.

Other investigators have also found depression to be a significant determinant of medication adherence in several disorders other than asthma. A recent meta-analysis of 31 studies (18,425 participants) of adults with various chronic conditions reported a 1.76 times greater odds for non-adherence in depressed patients [26]. Another report suggests that approximately 20 to 30 percent of prescriptions are never filled (primary non-adherence) and $50 \%$ of medications prescribed for chronic diseases are not taken as prescribed [2]. Medication non-adherence is associated with higher downstream health care costs [27], and can be reduced by improved self-management of chronic disorders such as asthma. We are unaware of any other study that has reported the effectiveness of asthma interventions on controller medication adherence among adolescents with depressive symptoms.

Other co-morbidities have been observed in asthma. Besides depressive symptoms, adolescents with asthma have also been found to have a higher prevalence of anxiety disorder [28] and internalizing behaviors [29]. These are linked through several psychological and biological factors such as the stress of asthma management, medication regimens, and avoidance of allergic triggers; or through cognitive responses to asthma symptoms such as learned helplessness or fear of bodily sensations. In the case of adolescents, having asthma symptoms may induce social anxiety (due to concern for negative evaluation by peers) that can significantly impact asthma-related outcomes [30].

The overall rate of controller medication use in this study was low (29\% at baseline) resulting in a small sample available for analyses. There are additional limitations to this study. First, we used self-reported measures of asthma controller medication adherence, which can have questionable validity and reliability [31,32]. We note that self-report of asthma controller medication adherence

Table 3 Comparison of controller medication adherence at 6 month follow-up by randomization group and by baseline elevated depressive symptoms, for students included in the analysis sample*

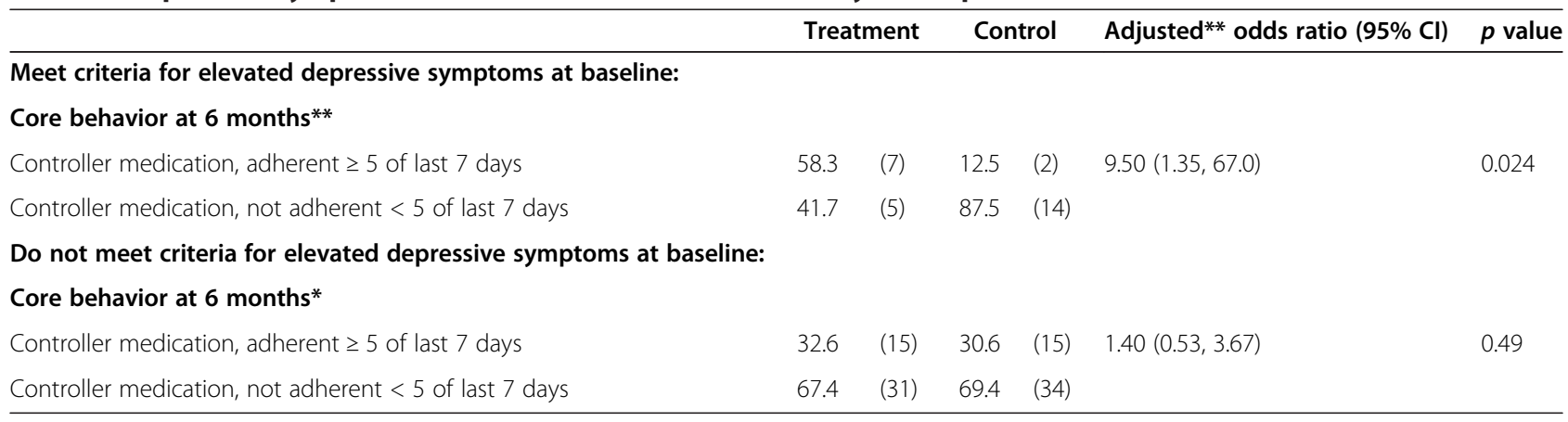

*Students enrolled in Puff City and reporting a controller medication at baseline assessment. ${ }^{*}$ Adjusted for controller medication adherence at start of intervention. 
has been used in national surveys such as National Health and Nutrition Examination Survey (NHANES) [33], and National Asthma Survey (NAS) [34]. Second, we cannot determine which component of the intervention was instrumental in motivating participants to be more adherent, i.e., depressed students received tailored messages about controller medication through the online program in addition to referrals made by the asthma referral coordinator for their depressive symptoms. Moreover, we cannot confirm that students followed up on referrals from the asthma referral coordinator and cannot report on the therapies or advice these students may or may not have received from these referrals. Consequently, we cannot speculate on the mechanism by which controller medication adherence was improved among students reporting depressive symptoms at baseline. Third, a sustained intervention effect for controller medication adherence post 6-month follow-up is unknown. Finally, because this study was done in urban adolescents with asthma, the results may only be applicable to other populations with characteristics similar to that of our study population. Given the limitations of this study, additional analyses in larger study samples are needed.

\section{Conclusions}

In these subgroup analyses of data from a RCT to evaluate an online asthma management program for urban adolescents with asthma, students who were randomized to the treatment group and met criteria for elevated depressive symptoms had better controller medication adherence when compared to a control group at 6-month follow up. Adolescents without depressive symptoms at baseline did not show statistically significant improvement in controller medication adherence. Interventions aimed at improving controller medication adherence as part of asthma self-management programs may need to be tailored for adolescents with depressive symptoms.

\section{Abbreviations \\ DPS: Diagnostic predictive scales; DISC: Diagnostic Interview Schedule for Children; aOR: adjusted odds ratio; ISAAC: International Study of Asthma and Allergies in Childhood; RCT: Randomized controlled trial; NHANES: National Health And Nutrition Examination Survey; NAS: National Asthma Survey; EPR: Expert panel report; HEDIS: Health plan Employer Data and Information Set; LHQ: Lung health questionnaire.}

\section{Competing interests}

There is no personal or financial support or author involvement with organization(s) with financial interest in the subject matter.

\section{Authors' contributions}

LG participated in discussion of analysis approach, results and interpretation, and prepared the manuscript. SLH conducted statistical analysis, discussed results and interpretation of analysis; reviewed manuscript. CC, is a co-investigator with input on study design and implementation, reviewed manuscript. DRO is a co-investigator with input on study design and implementation; reviewed manuscript. CLMJ is the Principal Investigator, participated in discussion of analysis approach and interpretation of results, assisted in preparation of manuscript and manuscript review. All the authors have read and approved the final manuscript.

\section{Research support funding}

This research was funded by the National Institutes of Health, National Heart, Lung, and Blood Institute. [Grant \# R01 HL67462-01].

\section{Author details}

'Pediatric Pulmonary Division, Department of Pediatrics, Children's Hospital of Michigan, Wayne State University School of Medicine, 3901 Beaubien St, Detroit, MI 48201, USA. ${ }^{2}$ Department of Public Health Sciences, Henry Ford Health System, Detroit, MI, USA. ${ }^{3}$ Clinical Allergy and Immunology, Georgia Health Sciences University, Augusta, GA, USA.

Received: 18 March 2013 Accepted: 30 October 2013

Published: 11 November 2013

\section{References}

1. Akinbami LJ MJ, Bailey C, Zahran HS, King M, Johnson CA, Liu X: Trends in asthma prevalence, health care use, and mortality in the United States, 2001-2010. In NCHS Data Brief, vol. 94. Hyattsville, MD: National Center for Health Statistics; 2012.

2. Sabate E: (Ed): Adherence to long-term therapies: evidence for action. Switzerland: World Health Organization; 2003.

3. Milgrom H, Bender B, Ackerson L, Bowry P, Smith B, Rand C: Noncompliance and treatment failure in children with asthma. J Allergy Clin Immunol 1996, 98:1051-1057.

4. Bender B, Zhang L: Negative affect, medication adherence, and asthma control in children. J Allergy Clin Immunol 2008, 122:490-495.

5. Naimi DR, Freedman TG, Ginsburg KR, Bogen D, Rand CS, Apter AJ: Adolescents and asthma: why bother with our meds? I Allergy Clin Immunol 2009, 123:1335-1341.

6. Desai M, Oppenheimer Jj: Medication adherence in the asthmatic child and adolescent. Curr Allergy Asthma Rep 2011, 11:454-464.

7. Katon W, Lozano P, Russo J, McCauley E, Richardson L, Bush T: The prevalence of DSM-IV anxiety and depressive disorders in youth with asthma compared with controls. J Adolesc Health 2007, 41:455-463.

8. Ortega AN, McQuaid EL, Canino G, Goodwin RD, Fritz GK: Comorbidity of asthma and anxiety and depression in Puerto Rican children. Psychosomatics 2004, 45:93-99.

9. Goodwin RD, Fergusson DM, Horwood L: Asthma and depressive and anxiety disorders among young persons in the community. Psychol Med 2004, 34:1465-1474.

10. Guglani L, Havstad SL, Johnson CC, Ownby DR, Joseph CL: Effect of depressive symptoms on asthma intervention in urban teens. Ann Allergy Asthma Immunol 2012, 109:237-242. e232.

11. Dempe C, Junger J, Hoppe S, Katzenberger ML, Moltner A, Ladwig KH, Herzog W, Schultz JH: Association of anxious and depressive symptoms with medication nonadherence in patients with stable coronary artery disease. J Psychosom Res 2013, 74:122-127.

12. Sjosten N, Nabi H, Westerlund H, Salo P, Oksanen T, Pentti J, Virtanen M, Kivimaki M, Vahtera J: Effect of depression onset on adherence to medication among hypertensive patients: a longitudinal modelling study. J Hypertens 2013, 31:1477-1484.

13. Tang HY, Sayers SL, Weissinger G, Riegel B: The role of depression in medication adherence among heart failure patients. Clin Nurs Res 2013. Epub ahead of print: April 2, 2013, doi: 10.1177/1054773813481801.

14. Joseph CL, Peterson E, Havstad S, Johnson CC, Hoerauf S, Stringer S, Gibson-Scipio W, Ownby DR, Elston-Lafata J, Pallonen U, et al: A web-based, tailored asthma management program for urban African-American high school students. Am J Respir Crit Care Med 2007, 175:888-895.

15. Joseph CL, Ownby DR, Havstad SL, Saltzgaber J, Considine S, Johnson D, Peterson E, Alexander G, Lu M, Gibson-Scipio W, et al: Evaluation of a web-based asthma management intervention program for urban teenagers: reaching the hard to reach. J Adolesc Health 2013, 52:419-426.

16. Joseph CL, Baptist AP, Stringer S, Havstad S, Ownby DR, Johnson CC, Williams LK, Peterson EL: Identifying students with self-report of asthma and respiratory symptoms in an urban, high school setting. $J$ Urban Health 2007, 84:60-69. 
17. Weiland SK, Bjorksten B, Brunekreef B, Cookson WO, von Mutius E, Strachan DP: Phase II of the international study of asthma and allergies in childhood (ISAAC II): rationale and methods. Eur Respir J 2004, 24:406-412.

18. NIH: Expert Panel Report 2: Guidelines for the Diagnosis and Management of Asthma. Bethesda: National Institutes of Health; 1997. NIH Publication No. 97-4051: NHLBI.

19. National Institutes of Health: Expert Panel Report 3: Guidelines for the Diagnosis and Management of Asthma. Washington DC: National Institute of Health; 2007. NHLBI ed., vol. NIH Publication No. 07-4051.

20. National Committee for Quality Assurance: Use of Appropriate Medications for People with Asthma: HEDIS 2002, Technical Specifications, Volume 2. Washington, DC: National Committee for Quality Assurance; 2001.

21. Lucas CP, Zhang H, Fisher PW, Shaffer D, Regier DA, Narrow WE, Bourdon K, Dulcan MK, Canino G, Rubio-Stipec M, et al: The DISC Predictive Scales (DPS): efficiently screening for diagnoses. J Am Acad Child Adolesc Psychiatry 2001, 40:443-449.

22. Shaffer D, Fisher P, Lucas CP, Dulcan MK, Schwab-Stone ME: NIMH diagnostic interview schedule for children version IV (NIMH DISC-IV): description, differences from previous versions, and reliability of some common diagnoses. J Am Acad Child Adolesc Psychiatry 2000, 39:28-38.

23. Cubo E, Velasco SS, Benito VD, Villaverde VA, Galin JM, Santidrian AM, Vicente JM, Guevara JC, Louis ED, Leon JB: Psychometric attributes of the DISC predictive scales. Clin Pract Epidemiol Ment Health 2010, 6:86-93.

24. Leung PW, Lucas CP, Hung SF, Kwong SL, Tang CP, Lee CC, Ho TP, Lieh-Mak F, Shaffer D: The test-retest reliability and screening efficiency of DISC predictive scales-version 4.32 (DPS-4.32) With chinese children/youths. Eur Child Adolesc Psychiatry 2005, 14:461-465.

25. Hosmer DW, Lemeshow S: Applied Logistic Regression. 2nd edition. New York: John Wiley and Sons; 2000.

26. Grenard JL, Munjas BA, Adams JL, Suttorp M, Maglione M, McGlynn EA, Gellad WF: Depression and medication adherence in the treatment of chronic diseases in the United States: a meta-analysis. J Gen Intern Med 2011, 26:1175-1182.

27. Bender BG, Rand C: Medication non-adherence and asthma treatment cost. Curr Opin Allergy Clin Immunol 2004, 4:191-195.

28. Goodwin RD: Asthma and anxiety disorders. Adv Psychosom Med 2003, 24:51-71.

29. Feldman JM, Ortega AN, McQuaid EL, Canino G: Comorbidity between asthma attacks and internalizing disorders among Puerto Rican children at one-year follow-up. Psychosomatics 2006, 47:333-339.

30. Bruzzese JM, Fisher PH, Lemp N, Warner CM: Asthma and social anxiety in adolescents. J Pediatr 2009, 155:398-403.

31. Jerant A, DiMatteo R, Arnsten J, Moore-Hill M, Franks P: Self-report adherence measures in chronic illness: retest reliability and predictive validity. Med Care 2008, 46:1134-1139.

32. Garfield S, Clifford S, Eliasson L, Barber N, Willson A: Suitability of measures of self-reported medication adherence for routine clinical use: a systematic review. BMC Med Res Methodol 2011, 11:149,

33. Kit $B K$, Simon $A E$, Ogden $C L$, Akinbami LJ: Trends in preventive asthma medication use among children and adolescents, 1988-2008. Pediatrics 2012, 129:62-69.

34. Crocker D, Brown C, Moolenaar R, Moorman J, Bailey C, Mannino D, Holguin F: Racial and ethnic disparities in asthma medication usage and health-care utilization: data from the national asthma survey. Chest 2009, 136:1063-1071.

doi:10.1186/1710-1492-9-45

Cite this article as: Guglani et al:: Exploring the impact of elevated depressive symptoms on the ability of a tailored asthma intervention to improve medication adherence among urban adolescents with asthma.

Allergy, Asthma \& Clinical Immunology 2013 9:45.

\section{Submit your next manuscript to BioMed Central and take full advantage of:}

- Convenient online submission

- Thorough peer review

- No space constraints or color figure charges

- Immediate publication on acceptance

- Inclusion in PubMed, CAS, Scopus and Google Scholar

- Research which is freely available for redistribution

Submit your manuscript at www.biomedcentral.com/submit 